\title{
Do we need a more inclusive model for understanding Ethics in palliative care in the developing countries?
}

\author{
Zubia Veqar* \\ Centre for Physiotherapy and Rehabilitation Sciences, Jamia Millia Islamia, New.Delhi
}

Received: December 10, 2016; Accepted: December 15, 2016; Published: December 21, 2016

*Corresponding author: Zubia Veqar, Assistant Professor in Physiotherapy, Centre for Physiotherapy and Rehabilitation Sciences, Jamia Millia Islamia, New.Delhi. E-mail: veqar.zubia@gmail.com

\section{Editorial}

"Death is not extinguishing the light; it is putting out the lamp because the dawn has come." - Rabindranath Tagore

Palliative care aims at providing relief in every possible manner to the patient as well as care giver. Research has substantiated the idea that Palliative care is not only end of life care. It has been proved that appropriate treatment not only leads to improvement in life expectancy and quality of life but it also leads to decreased health care costs. Palliative care is a team work where there is an integration of expertise of professionals from various disciplines to manage the complex needs of end of life care. The team typically includes professionals from medicine, nursing, social work, physiotherapy with additional support from other fields like psychology as and when required [1-2].

A sound knowledge of medical ethics is essential for transparency and understanding of decision making as well as conflict management [3] .The latest guidelines of bioethics available are the revision of the Declaration of Helsinki, which was adopted in 2013[4]. The roots of these guidelines of modern medical ethics can be traced back to "The Nuremberg code" which was established in the immediate aftermath of the Second World War [5]. There are four principles of medical ethics: autonomy, beneficence, non-malfeasance and justice [6-7].Autonomy empowers the patient. The responsibility of making the patient informed about the condition lies with the consultant. This information makes the patient an integral part of the health care decision making but in certain conditions where the patient's intellect is compromised the care takers take this role. This principle becomes especially difficult to follow when the prognosis is poor. Beneficence and Maleficence are inter relate principles as they have to be appropriately balanced for appropriate decision making. Justice is about the availability of health care. The issue of dignity, truthfulness and honesty are the other additional important principles in this regard. Based on these existing principles and with some new editions keeping the cultural, racial and other differences in mind some of the countries like Australia [8] have developed their own guidelines.
The movement of palliative care is fairly new in the developing countries like India, Pakistan and some Middle Eastern countries [9-12].A mapping project was commissioned by the Worldwide Palliative care Alliance to present the development of palliative care services in the world. The report placed Afghanistan, Bhutan and Maldives in group 1(no known activity), Pakistan, Bangladesh and Sri Lanka in Group 3a (isolated palliative care provision) and India and Nepal in Group 3b (generalized palliative care provision) [13]. These countries have their own set of barriers like poverty and lack of education, awareness and resources. Lack of awareness among professionals, administrators, educators and the public along with narcotic regulation also add to the list of barriers [9] At the same time they also come with the unique strength associated with an intact family structure and values and spiritual lifestyle [13-14]. Because of the uniqueness of the ethical dilemmas and issues of the region it is difficult to explain them with the western model of palliative care ethics.

Autonomy in this context comes with its own understanding. Firstly the decision making is not done by the patient alone. This is a task which involves the family members as well as the physician [14]:Secondly the physician is considered next to God [15]. On one side the decision making becomes easier because it should only be guided by the sound medical knowledge whereas on the other side the responsibility increases because the care giver looks up to the physician for a magical solution to save the patient's life. This situation is the two sides of the same coin. Thirdly family plays an integral role in all aspects of the plan of management right from diagnosis to treatment. A number of times care givers do not want the patient to know about the details of the condition or sometimes even the diagnosis itself [16]. Hence the concept of confidentiality is not completely maintained either. This in itself raises important concerns and issues for the Palliative care team.

Beneficence and Maleficence become difficult to apply as far as pain management is concerned. There are unrealistic legal restrictions in availability of opiates and there are a number of 
myths associated with drug dependence in the general public. This has made pain management a very difficult issue. This problem has been further exaggerated by the lack of awareness amongst professionals regarding pain associated with end of life $[9,10,11]$. Another aspect is home care in the traditional societies where the family and the patient would prefer home care over hospitalization. This leads to a decrease in quality of care which could be imparted otherwise. But it also decreases the load over the already overburdened health care services especially wherever specialty palliative care services are not available $[9,17]$.

The principle of Justice also becomes relative. The resources in these countries are limited and have to be distributed over a larger population. Therefore the term 'fair management' can be used. The costs of the management also need to be assessed in comparison with the outcomes. Clinical decision making becomes more difficult if the patent as well as the service provider has limited resources [16].Fewer research in the area is also responsible for the paucity of guidelines and evidence based practice.

Directive or guidelines based on understanding of death and dying, which is influenced by traditional value, culture and belief systems, seems to be more apt to the situation and easier to apply as well as to understand. The guidelines issued by Indian Society of Critical Care Medicine (ISCCM) and the Indian Association of Palliative Care (IAPC) are definitely a major step towards the establishing of the ethical guidelines [11] in India. Similar steps are required by other concerned organizations of other countries too. An inclusive model where religious and spiritual sensibilities are also integrated in the system would make its application better and wider.

\section{References}

1. Doyle D, Getting started guideline and suggestions for those starting a hospice/palliative care service. IAHPC press Houston 20092 edition [cited 2012 April 10]

2. Kelley AS, Meier DE. Palliative care-a shifting paradigm. New England Journal of Medicine. 2010;363(8):781-782. DOI: 10.1056/ NEJMe1004139.

3. Fetters MD, Churchill L, Danis M. Conflict resolution at the end of life. Crit Care Med. 2001;29(5):921-925.
4. Muthuswamy V, Sarin A, Pai SA et al. The new 2013 seventh version of the Declaration of Helsinki-more old wine in a new bottle? Indian J Med Ethics. 2014;11(1):2-4.

5. Thierena M, Mauronb A. Nuremberg code turns 60.Bulletin of the World Health Organization. 2007;85 (8): 573-574.

6. Mohanti, BidhuK.. Ethics in palliative care. Indian Journal of Palliative Care . 2009;15(2):89-92.

7. Gwyther Liz. Ethics and palliative care. Continuing Medical Education. 2011;29(7):274-276.

8. National Health and Medical Research Council. An ethical framework for integrating palliative care principles into the management of advanced chronic or terminal conditions. National Health and Medical Research Council. 2011. 1-27.

9. Rajagopal MR. The current status of palliative care in India. In Magrath I, editor. Cancer Control 2015: Cancer Care in Emerging Health Systems. Brussels: INCTR; 2015.

10.Veqar Z. The perspectives on including palliative care in the Indian undergraduate physiotherapy curriculum. Journal of clinical and diagnostic research. JCDR. 2013;7(4):782-787.

11. Myatra SN, Salins N, Iyer S et al End-of-life care policy: An integrated care plan for the dying. Indian J Crit Care Med. 2014;18:615-635.

12. Mudigonda T, Mudigonda P. Palliative cancer care ethics: Principles and challenges in the Indian setting. Indian J Palliat Care. 2010;16(3):107-110.

13. Lynch T, Connor S, Clark D. Mapping levels of palliative care development: a global update. J Pain Symptom Manage. 2013;45(6):1094-1106.

14. Sharma H, Jagdish V, Anusha P, Bharti S. End-of-life care: Indian perspective. Indian J psychiatry. 2013;55(Suppl 2):S293-S298. doi:10.4103/0019-5545.105554.

15. Aggarwal KK. Blog.kkaggarwal.com.[cited 072016 Dec 07].

16. Chandra PS, Chaturvedi SK, Kumar A, et al. Awareness of diagnosis and psychiatric morbidity among cancer patients: A study from South India. J Psychosom Res 1998;45:257-261.

17. Chaturvedi SK. Ethical dilemmas in palliative care in traditional developing societies, with special reference to the Indian setting. Journal of medical ethics. 2008;34(8):611-615. doi:10.1136/ jme.2006.018887. 